\title{
Research on the Relationship between Marketing Strategy and Brand Development of Apparel Companies Based on Data Mining Technology
}

\author{
Yinan $\operatorname{Sun}^{1, *}$ \\ ${ }^{1}$ DUFE, Surrey International Institute, 116025, Dalian, China
}

\begin{abstract}
The apparel industry is an important part of China's economy and an important industry that drives the continuous improvement of national living standards. As the size of China's internal market continues to expand, competition in the apparel industry has become increasingly fierce, making the development of apparel companies face huge challenges. For apparel companies, establishing a brand style that is unique and effective in attracting consumers, along with a sound brand marketing strategy, is a must for apparel companies to survive. This paper takes Company X, which is engaged in the apparel industry, as an example, and firstly analyses Company $\mathrm{X}^{\prime}$ s brand marketing, then introduces data mining technology to analyse the relationship between Company X's marketing strategy and brand development in view of the shortcomings of Company X's brand marketing, and finally proposes a corresponding brand marketing strategy in order to provide a reference for the formulation of Company X's brand marketing plan and promote the development of the apparel industry.
\end{abstract}

\section{Introduction}

As China's comprehensive national power continues to rise and the social and economic level continues to improve, people's demand for clothing is also showing a diversified trend, where consumers' preference for clothing brands is often an important factor in determining consumer consumption. In the face of the complex and changing market environment, formulating a good marketing strategy, creating personalized brand qualities and precisely meeting the personalized requirements of the target group is the way to the development of the current apparel enterprises. And to determine an effective marketing strategy, we must determine the relationship between marketing strategy and brand development, targeted screening travel and effective marketing tools and marketing positioning, which in turn can make the enterprise brand to obtain long-term development [1-2].

And with China's vigorous support for the Internet, the garment industry is gradually on par with high-tech industries. The garment industry can only keep up with the trend of the times and come out of a unique development path to enhance its core competitiveness by continuously learning the development model of emerging industries, deepening the combination with mobile Internet, cloud computing, big data and Internet of Things, introducing the latest information technology and using it to formulate various strategies for enterprise development. The development and growth of ecommerce, industrial Internet and Internet finance can bring significant changes to the development of traditional garment enterprises, and have a significant role in promoting the transformation and upgrading of garment enterprises [3-5].

\section{Analysis of Company X's business status and marketing strategy}

\subsection{Company $X$ 's business status}

Company $\mathrm{X}$ is a large apparel company in China. The company's apparel marketing business operates primarily through partnerships with independent retailers. The retailers each own and operate their own stores, and tailor their concepts and services to local needs. There is also extensive collaboration in expansion, sourcing, logistics, IT and marketing communications to achieve economies of scale. The business takes different designs for clothing and sells different brands, and stocks all brands in its stores. Company $\mathrm{X}$ is further developing and developing its brand-related commitments, with key initiatives including the development of customer loyalty programs and the development of corresponding private label brands, creating more value for customers with more unique brand collections. Brand development is therefore one of X's strategic themes.

However, in the current apparel market, Company $\mathrm{X}$ is facing fierce competition in apparel. The competitors in the industry are roughly divided into two types for analysis: The first type is the local apparel companies in China, mainly from independent apparel design brands.

*Corresponding author: sunyinan1314@icloud.com 
Local Swedish apparel has the advantage of channels for easy promotion and easy acceptance by local consumers. The other type is the clothing brands imported from abroad, which have a high reputation and therefore a competitive price. The current competitiveness of the existing competitors in the Chinese going-to-wear industry is extremely strong, and it will take considerable effort for Company $\mathrm{X}$ to stand out from the competition in order to gain a certain market share.

\subsection{Company X's existing brand marketing strategy}

\subsubsection{Product strategy and shortcomings}

The apparel industry needs to launch new products periodically according to the season, and Company X adopts a product strategy to keep up with the trend by ensuring the high quality of the basic models of each season, while adopting a seasonal development strategy for other styles. Company X puts the quality of its clothing first and does not compromise quality even in the face of high cost pressure, but uses economies of scale to improve the brand's cost performance, which is recognized by consumers. Company $\mathrm{X}$ 's clothing is mainly divided into spring and summer clothing and fall and winter clothing, and there are four categories: casual clothing, sportswear, women's clothing and baby clothing, with baby clothing accounting for slightly more than $30 \%$ of the total. In order to meet the needs of customers for one-stop shopping, Company A has a full range of products in each store.

However, Company $\mathrm{X}$ does not have a clear market positioning and favours basic models, which makes the brand not differentiated, and is not strong enough to shape the clothing brand and enhance the core competitiveness and grade of clothing products through accurate positioning. Meanwhile, Company X's ability to respond quickly to the market is weak, and the production cycle of the entire apparel supply chain is about 6 months, during which time fashion trends may change significantly, resulting in serious brand stagnation.

\subsubsection{Price strategy and shortcomings}

Setting attractive prices is an effective means for companies to attract consumers, and reasonable and strong pricing is especially important in the face of fierce competition in the same industry and disruptive crossborder competition. $x$ The company's four main products adopt the sales model of same store sales, and need to adopt differentiated pricing strategies for different product categories. Among them, casual wear has a simple process and adopts a low price strategy; baby wear has a wide range of categories, pursues mass production and requires high quality process, and adopts medium price pricing; sportswear focuses on functionality, carries more added value and has higher pricing, which is the main profit point of the company.
Currently, Company X's branded apparel is still set at a low to mid-range level standard, and Company $\mathrm{X}$ mainly adopts a low-price strategy to quickly penetrate the apparel market. Due to its pursuit of quality, the overall cost is still relatively high. However, the current global trend of apparel consumption is younger, more styles and lower prices are the main development trend, and higher pricing will inevitably affect the purchase choice of most consumers. At the same time, Company $\mathrm{X}$ is very conservative in regulating clothing prices, preferring the cost pricing method, but in the face of other brands' low price promotions, combination price cuts, or some new entrants occupying the market regardless of cost, Company $\mathrm{X}$ fails to adopt a diversified price strategy for defence, and the price fluctuation ratio is too low. This led to a large number of consumers choosing other brands with strong promotions.

\subsubsection{Channel strategy and shortcomings}

Company X's channel strategy is based on Company A's business operating in partnership with independent retailers who each own and operate their own stores and tailor their concepts and services to local needs. The specific process that is when the company's apparel products are directly responsible for the warehousing and distribution of goods by the relevant departments.

But in today's information explosion, the importance of e-commerce in the present day can not be overstated. All channel construction in the past was dependent on traditional stores, for which management has become path dependent. Not enough attention has been paid to the changes brought by the Internet. Despite the rapid growth of online sales, Company $\mathrm{X}$ has not yet established an online apparel sales channel to run its apparel business, missing the opportunity of channel change brought by the Internet and the timely communication and interaction with consumers.

\subsubsection{Promotional strategies}

Research shows that even if consumers have already thought of several alternative brands before shopping, their final purchase is still largely influenced by the terminal promotion. Effective promotion can provide product information, showcase product selling points, and more importantly, make consumers feel that they are getting more value for their money. Currently, Company $\mathrm{X}$ advertises in newspapers and on TV, lays out posters in stores, and provides brochures to raise the attention of target consumers to the brand and promotions. In addition, Company X's retail stores offer discounts on some of the less popular styles or out-of-season styles. During the holiday season. The retail stores also conduct holiday promotions on some clothing styles.

However, the emergence of mobile Internet makes Company X's promotional communication channels misplaced, unable to communicate and interact with young consumers, and the brand value and product information cannot be effectively and quickly delivered to the target consumer groups. At the same time, 
Company $\mathrm{X}$ did not base on product characteristics and ignored the stimulating effect of promotion on consumers' purchase only from the profit index, as Company A's baby clothes accounted for $30 \%$ of the overall sales, and the buyers of baby clothes were mainly female consumers, and most of those who often went to supermarkets for "one-stop shopping" were female consumers, who were most sensitive to the terminal Company X seldom adopts discount strategy to capture the psychology of this mainstream consumer group, and gives up this customer group to the competitor with hot promotion activities, so Company $\mathrm{X}$ urgently needs new marketing ideas to cope with the unfavorable situation of clothing sales at present.

\section{Data analysis of company x's marketing market}

\subsection{Marketing Data Mining System}

In order to analyse the relationship between the marketing strategy and brand development of Company $\mathrm{X}$, it is necessary to focus on the factors that influence the marketing of different brands of the company, so as to determine the market positioning of the brand and ultimately help to target the development of marketing strategies that are beneficial to the brand. Therefore, this paper introduces a data mining system to deeply explore the brand marketing influencing factors of Company $\mathrm{X}$. The specific architecture of the system is shown in Fig. 1.

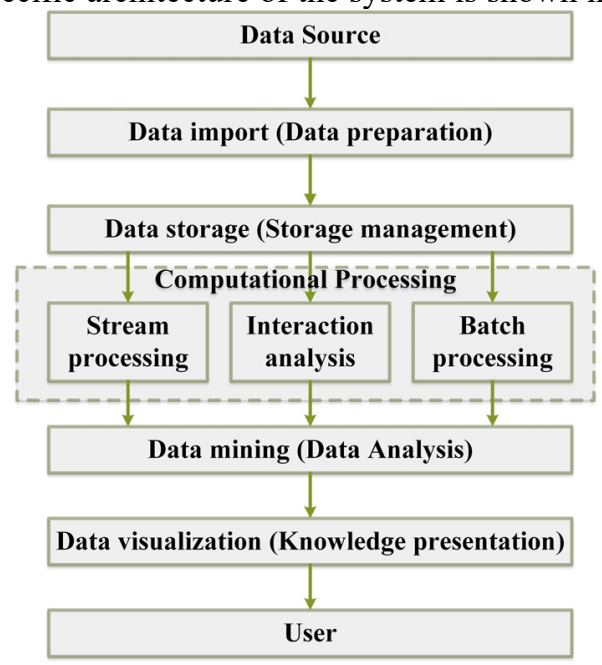

Fig.1 The specific architecture of the data mining system

Data source means the source of data, and in the era of big data, data is more often derived from the Internet, Internet of Things and stored data of enterprises. Data preparation refers to the simple processing of data, including import, extraction, conversion and loading, and the processed data can be used to prepare for data storage, calculation and analysis. Data storage management refers to the storage and management of data. Big data involves a large amount of data and a complex structure. When storing a large amount of data, it is necessary to ensure that it is within the operating range of the machine and to support the requirements of unstructured data management when the stored data needs to be called for computation and analysis. Therefore, the main problem facing big data storage is to store large amounts of data at very low cost and also to accommodate the management requirements of unstructured data. Computational processing is the selection of the right algorithm model to process data quickly according to the type of data and analysis objectives. It mainly includes three aspects: batch processing, stream processing and interactive analysis. The traditional model of massive data processing is difficult to adapt to the needs of the big data era, and thus the mainstream computing architecture adopts distributed computing. Data analysis, which refers to the mining of valuable knowledge from the sea of massive and disorganized data, is the key to big data mining. The purpose of data analysis is to prepare the data for enterprise application. Knowledge presentation, which can be understood as data visualization, shows the mined results to users in an intuitive and visual way.

In general, the technical framework of big data has four main parts: data collection, data storage, data analysis and data application. Firstly, enterprises should collect data, store them in their own data warehouse through ETL software, and then use algorithmic models to analyse the data. data warehouse, then use algorithmic models to explore the potential value of the data, and finally apply the valuable information to design, production or sales.

For apparel companies, they should make full use of the technical framework of big data to deal with their own internal existing data, while collecting external data, and on the basis of making full use of the data, they should segment consumers by building a consumer database to gain insight into consumer needs, and provide personalized services to consumers based on the value of the data mined, while combining their own actual situation from a marketing perspective.

\subsection{Relationship analysis}

According to the analysis results of the data mining system, it can be seen that the main influencing factors of Company X's brand marketing are climate, season, gender, age group, price and style.

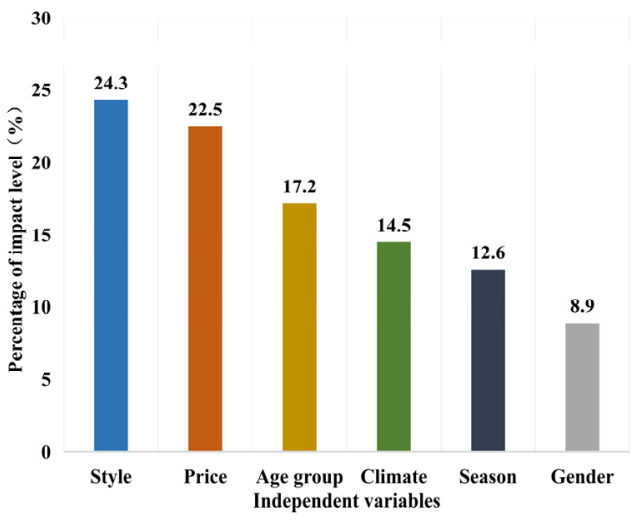

Fig.2 Influence degree of marketing factors 
With the development of the times, consumers in the Chinese market have gradually improved their taste in life, paying attention to the matching of clothing and identity and temperament. The diversified development of culture makes different types of people have customized and personalized demand for clothing. Company X needs to segment the entire apparel market, select its main market for different target groups, and deliver its differentiated value to the target market. Therefore, climate, season, gender, age group, price and style were used as independent variables, and sales were used as dependent variables, and the data was imported into the data mining system to analyse the degree of influence of different on the final sales when they were used as brand staples.

From Figure 2, it can be seen that the factors affecting the marketing effect in order of importance are style, price, age group, climate, season and gender. With the development of the times, consumers in the Chinese market have gradually improved their taste in life and pay attention to the matching of clothing and identity and temperament. The diversified development of culture makes different types of people have customized and personalized demand for clothing. the first thing Company $\mathrm{X}$ has to do is to do a good job of style design and deliver its differentiated value to the target market. Secondly, since there is a huge potential in the middle and low-end market in China, Company X's brand clothing should focus on good quality and inexpensive middle and low-end clothing, which is a good choice for the existing Chinese market consumers. Then $\mathrm{X}$ company should mainly focus on baby clothes and casual clothes as the main target, and take young consumers and family consumers as the target group, and deliver the differentiated value of $\mathrm{X}$ company's clothing brand to their brain. Finally, Company X can design different styles of men's and women's clothing according to the climate and seasons. From the above analysis, it is clear that different main factors in the marketing strategy will affect the final development of the brand, so the main factors of marketing should be determined according to the actual situation of the company.

\section{Suggestions for improvement of marketing strategy}

In view of the shortcomings of the marketing strategy of the existing brand of Company $\mathrm{X}$, this paper gives some suggestions for improvement of brand marketing as follows.

(1) Improve clothing brand awareness. company X's management team needs to clarify the importance of brand building, find a marketing mix strategy that suits the specific environment at the moment, adopt a multibrand strategy to maintain price advantages in order to occupy a certain market share, and at the same time pay attention to clothing design, design some personalized clothing independently based on reference to fashion trends, and build brand influence.
(2) Pricing according to market demand and ability. Company $\mathrm{X}$ in the pricing process, too much consideration of the cost, the acceptance of the target group, the lack of awareness of psychological perception, the result is that consumers generally feel that company $\mathrm{X}$ clothing lack of price attractiveness, this impression has been formed, even if there is a cost-effective single product appear, consumer psychological perception is not obvious. It is recommended that Company $\mathrm{X}$ develops a pricing strategy that is more in line with consumer psychology based on market demand.

(3) Accurately grasp consumer psychology and purchasing behaviour. At this stage, Company $\mathrm{X}$ ignores the preferences of young consumers, over-considers the company's own economic interests, and has poor communication channels with consumers, leaving consumers with emotions without communication channels. company A can strengthen the construction of consumer communication, and reasonably solve consumer problems can make negative emotions into positive behaviour.

\section{Conclusion}

Taking Company $\mathrm{X}$ as an example, this paper analyzes the current situation and shortcomings of Company X's marketing strategy, then introduces a data mining system to study the six decisive influencing factors in the marketing process for brand development, and determines the importance of the influencing factors for the final impact results, and thoroughly studies the relationship between Company X's marketing strategy and brand development. In view of the shortcomings of the existing influencing marketing strategies of Company X, corresponding improvement suggestions are proposed in order to provide reference for the development of Company X.

\section{References}

1. Ruan Yan-wen, Gu Li-wen, Liu Xiao-gang and He Zhong-yu, "The study on the brand earnings' features of growth corporate brand: Based on an empirical analysis in apparel industry," 2016 13th International Conference on Service Systems and Service Management (ICSSSM), Kunming, China, 2016, pp. 1-6.

2. L. Jiang and K. Zhang, "Apparel brand overlap based on customer perceived value and eye-tracking technology," in Tsinghua Science and Technology, vol. 23, no. 1, pp. 47-64, Feb. 2018.

3. S. H. K. Nallapaneni and S. Babu, "Use of Social Media by Apparel Brands in India," 2018 International Conference on Advances in Computing, Communications and Informatics (ICACCI), Bangalore, India, 2018, pp. 2005-2010.

4. Li Quan-ming, Zhu Yi and Xiao Xuan, "A business opportunity in the e-era: Apparel network marketing," MSIE 2011, Harbin, China, 2011, pp. 48-51. 
5. X. Qi and W. Zhang, "Study on apparel marketing model based on IPv6," 2010 IEEE International Conference on Software Engineering and Service Sciences, Beijing, China, 2010, pp. 438-441. 